substitutions have the same composition formula as linear fractional substitutions. Hence, according as -1 is a square or a not.square, $H^{\prime}$ is simply isomorphic to the "real" or the "imaginary" form * of the group of linear fractional substitutions of determinant unity. Thus, for $p^{n}>3, H^{\prime}$ is simple.

15. Observing that the squares of the substitutions

$$
O_{1,2}^{a, \beta}, \quad O_{1,2}^{a, \beta} T_{13} C_{1} C_{2} C_{3}, \quad O_{1,2}^{a, \beta} T_{13} T_{24}
$$

are respectively $Q_{1,2}^{\alpha,-\beta}, \quad O_{1,2}^{\alpha, \beta} O_{3,2}^{\alpha, \beta}, \quad O_{1,2}^{\alpha, \beta} O_{3,4}^{\alpha, \beta}$, we may unite our results into the following

TheOREM : The squares of the linear substitutions on $m$ indices in the $G F\left[p^{n}\right], p \neq 2$, which leave invariant the sum of the squares of the $m$ indices, generate a group, which for $m=2 k+1$ has the order

$$
\frac{1}{2}\left(p^{2 n k}-1\right) p^{2 n k-n}\left(p^{2 n k-2 n}-1\right) p^{2 n k-3 n} \cdots\left(p^{2 n}-1\right) p^{n}
$$

and is simple except when $p^{n}=3, m=3$; while for $m=2 k>4$ it has the factors of composition 2 and

$$
\frac{1}{4}\left[p^{n k}-( \pm 1)^{k}\right] p^{n k-n}\left(p^{2 n k-2 n}-1\right) p^{2 n k-3 n} \cdots\left(p^{2 n}-1\right) p^{n} \text {, }
$$

the sign \pm depending upon the form $4 l \pm 1$ of $p^{n}$.

UNIVERSITY OF CALIFORNIA,

February 10, 1898.

\title{
A PROOF OF THE THEOREM :
}

$$
\frac{\partial^{2} u}{\partial x \partial y}=\frac{\partial^{2} u}{\partial y \partial x}
$$

BY MR. J. K. WHITTEMORE.

(Read before the American Mathematical Society at the Meeting of April $30,1898$.

THEOREm : Let $u=f(x, y)$ denote a function of the two independent variables $x$ and $y$ which, together with its first derivatives and the two second derivatives in question, is continuous in the neighborhood of the point $(x, y)$; then $\frac{\partial^{2} u}{\partial x \partial y}=\frac{\partial^{2} u}{\partial y \partial x}$

$$
\text { Let } \frac{\partial^{2} f(x, y)}{\partial x \partial y} \text { denote } \frac{\partial}{\partial x}\left(\frac{\partial f(x, y)}{\partial y}\right)
$$

* Moore : Mathematical Papers of the Chicago Congress (1893), "A doubly-infinite system of simple groups," $\& \& 5-6$. 
and let $\frac{\partial^{2} f(x, y)}{\partial y \partial x}$ denote $\frac{\partial}{\partial y}\left(\frac{\partial f(x, y)}{\partial x}\right)$.

Let $\left(x_{0}, y_{0}\right)$ be any point for which the conditions of the theorem are fulfilled and let the lines $x=a, x=b, y=c$, $y=d$ bound a region of the plane enclosing the point $\left(x_{0}, y_{0}\right)$ and so small that the conditions stated are satisfied throughout the interior of the rectangle and on its boundary. Under these conditions we have

$$
\begin{aligned}
& \int_{c}^{d} d y \int_{a}^{b} d x \frac{\partial^{2} f(x, y)}{\partial x \partial y}=f(b, d)-f(b, c)-f(a, d)+f(a, c), \\
& \int_{a}^{b} d x \int_{c}^{d} d y \frac{\partial^{2} f(x, y)}{\partial y \partial x}=f(b, d)-f(a, d)-f(b, c)+f(a, c) .
\end{aligned}
$$

But, under the conditions of the theorem,

$$
\int_{a}^{b} d x \int_{c}^{d} d y \frac{\partial^{2} f(x, y)}{\partial y \partial x}=\int_{c}^{d} d y \int_{a}^{b} d x \frac{\partial^{2} f(x, y)}{\partial y \partial x}
$$

Hence $\quad \int_{c}^{d} d y \int_{a}^{b} d x\left(\frac{\partial^{2} f(x, y)}{\partial x \partial y}-\frac{\partial^{2} f(x, y)}{\partial y \partial x}\right)=0$.

Now, if a function, continuous in the neighborhood of a point $\left(x_{0}, y_{0}\right)$, is such that its integral, extended over any rectangle enclosing this point, is zero, it is readily seen that the function cannot be positive or negative at the point $\left(x_{0}, y_{0}\right)$. Hence

$$
\frac{\partial^{2} f(x, y)}{\partial x \partial y}-\frac{\partial^{2} f(x, y)}{\partial y \partial x}=0
$$

at the point $\left(x_{0}, y_{0}\right)$. But this was any point, and the theorem is proved.

\section{SOME OBSERVATIONS ON THE MODERN THEORY OF POINT GROUPS.}

BY MISS FRANCES HARDCASTLE.

The origins of the theory of point groups are to be found in Brill and Noether's classic memoir (see infra) published nearly twenty-five years ago, but it is only within the last fifteen years that systematic attention has been given to the subject by the Italian mathematicians, Segre, Bertini, 